\title{
Iliac Endoconduit: Application in Unfavorable Iliac Anatomy to Facilitate Emergency Abdominal Aortic Aneurysm Repair
}

\author{
Thomas Aherne ${ }^{\mathrm{a}, \mathrm{b}}$, Khairun I. Abdul-Jalil ${ }^{\mathrm{a}}$, Zahraa Al-Hilli ${ }^{\mathrm{a}}$, Peter A. Naughton ${ }^{\mathrm{a}}$
}

\begin{abstract}
Extensive occlusive ilio-femoral arterial disease may be considered a contraindication to endovascular aortic aneurysm repair. Recent technical innovations describe the use of an "internal endoconduit" to navigate challenging iliac anatomy. Internal endoconduit is a technique in which "controlled rupture" of the external iliac artery using a covered stent allows controlled trans-femoral passage of the delivery sheath for endovascular aneurysm repair (EVAR). Here, we present two cases where iliac endoconduit was used to overcome unfavorable iliac artery anatomy in the setting of an emergency EVAR.
\end{abstract}

Keywords: Endoconduit; EVAR; Rupture

\section{Introduction}

Endovascular aneurysm repair (EVAR) in the emergency setting is now the standard of care in many tertiary centers and is associated with a significant reduction in 30-day mortality when compared with open repair [1].

Nevertheless, stenotic iliac anatomy often limits endovascular stent introduction exposing the patient to the increased morbidity of open surgery $[2,3]$. Innovative iliac access techniques such as arterial endoconduits [4] aim to optimize stent graft access.

The use of iliac endoconduit (IE) in the emergency setting is infrequently described. Here, we describe two cases where IE was used to overcome unfavorable iliac artery anatomy in the setting of an emergency EVAR.

\section{Case Reports}

\section{Case 1}

A 65-year-old male presented to a peripheral institution with left

\footnotetext{
Manuscript accepted for publication November 18, 2016

aDepartment of Vascular Surgery, Beaumont Hospital, Dublin 9, Ireland ${ }^{\mathrm{b} C}$ Corresponding Author: Thomas Aherne, Beaumont Hospital, Dublin 9, Ireland. Email: thomasaherne@rcsi.ie
}

flank discomfort. His medical history included ischemic heart disease with recurrent unstable angina, atrial fibrillation and peripheral arterial disease. Contrast-enhanced computed tomography (CT) revealed an infra-renal abdominal aortic aneurysm (AAA) with an associated retroperitoneal hematoma. The aneurysm measured $5.5 \mathrm{~cm}$ in maximum diameter with marked vascular calcification. The diameter of left external iliac artery (EIA) was $4 \mathrm{~mm}$ as a result of circumferential calcification while the right common iliac artery (CIA) was occluded. He was urgently transferred to a tertiary vascular center for repair of his ruptured AAA.

On arrival, the patient was transferred to the operating theatre. His significant cardiac history rendered an open approach to be of very high risk. As such, following informed consent, an endovascular repair under local anesthesia was deemed the most appropriate intervention. A standard left common femoral artery cut-down was performed. In view of his occluded right CIA, a second arterial access was obtained via the left brachial artery to facilitate intraoperative angiography (Fig. 1). Initial left-sided serial dilatation of the EIA with a 5, 6 and 8-mm balloon was undertaken; however, stent graft delivery to the aorta was unsuccessful. Subsequently, an internal endoconduit was fashioned using two stent grafts. Both a $93 \mathrm{~mm}$ (long) by $16 \mathrm{~mm}$ (proximal diameter) by $10 \mathrm{~mm}$ (distal diameter)

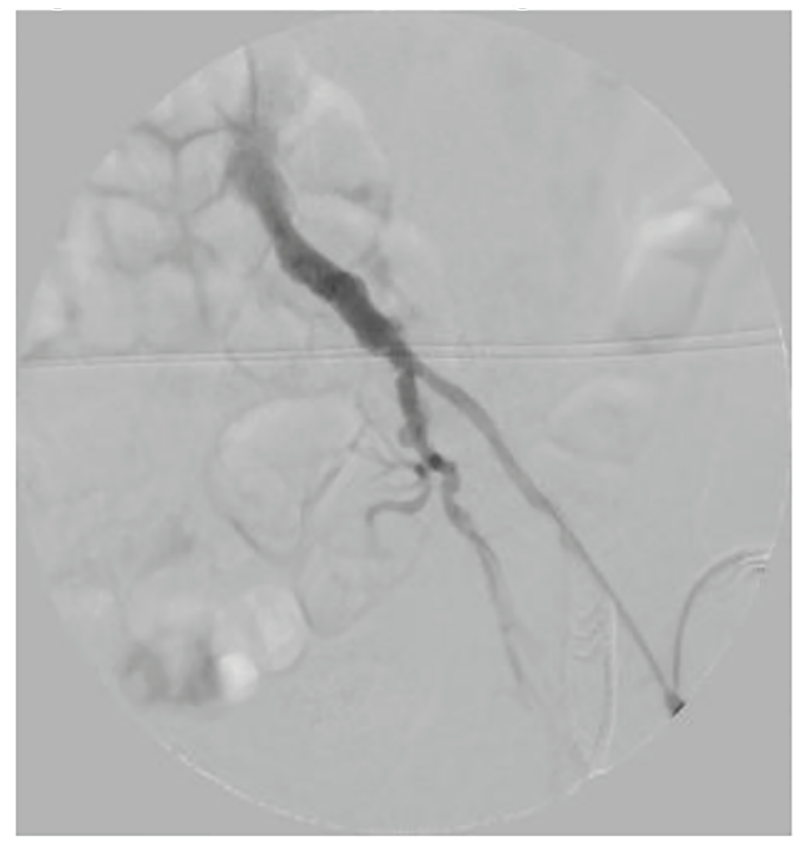

Figure 1. Narrowed left iliac segment. 


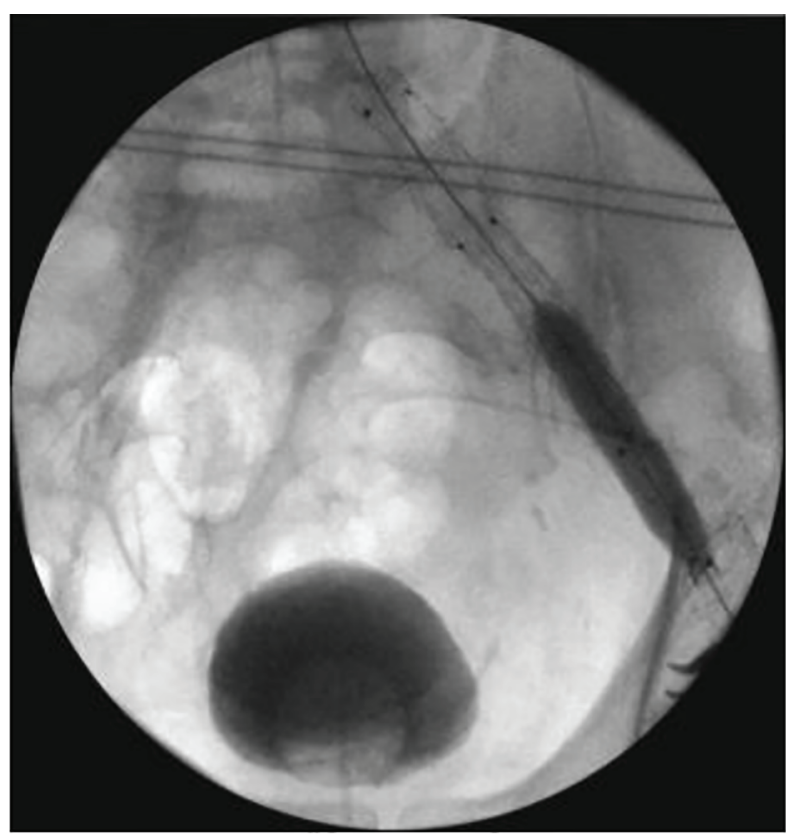

Figure 2. Conduit dilatation.

(16-10-93) and a 10-10-82 Endurant II stent graft (Medtronic, Hertfordshire, UK) were placed from the proximal CIA extending to the distal left EIA. Once deployed, the endoconduit was dilated using $10 \mathrm{~mm}$ balloon (Fig. 2). This allowed delivery of the main body aorto-uni-iliac (28-14-108) and a bridging 16-16-82 Endurant II stent graft, which were successfully deployed. A femoro-femoral bypass was deferred at this point as the patient had well perfused peripheries due to the presence of pre-existing collateralization. The postoperative course was unremarkable. The patient was discharged from the hospital on day 7 postoperatively.

\section{Case 2}

The second case was a 72-year-old female who presented to the emergency department with left-sided abdominal pain, a tender, palpable AAA and a temperature of $38.5^{\circ} \mathrm{C}$. She was a heavy smoker with medical history including ischemic heart disease, non-insulin dependent diabetes mellitus and congestive cardiac failure with an ejection fraction of $20 \%$. A CT angiogram of the aorta revealed a saccular $4.9 \mathrm{~cm}$ infrarenal AAA with periaortic fat stranding, a stenotic left iliac system (Fig. 3) and an occluded right CIA. A clinical and radiological diagnosis of mycotic aneurysm was established following multi-disciplinary discussion despite negative blood cultures. Overnight, despite broad-spectrum antibiotic cover, her pain worsened and following discussion with the patient and family, a decision to perform emergency EVAR was taken due to the risk of imminent rupture. Open options were not considered due to significant medical co-morbidities.

A similar operative technique as previously described was utilized; however, brachial access was not secured due to an inadequate caliber left-sided vessel (3 mm) (Fig. 3). An IE

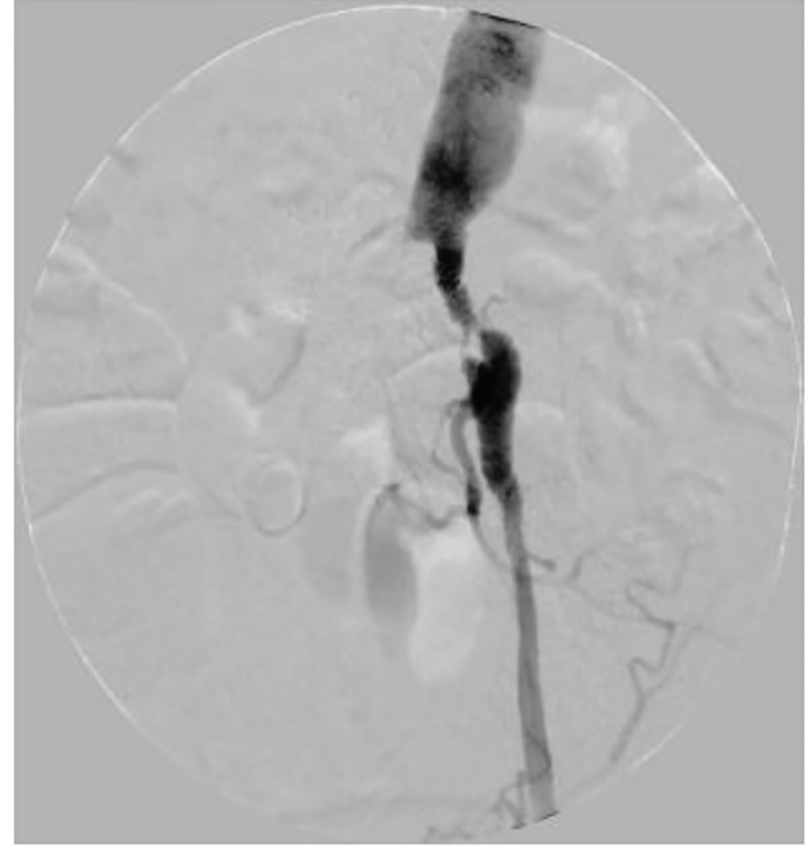

Figure 3. Stenotic left iliac system.

was fashioned using a 16-10-124 Endurant II stent graft and placed in the proximal left CIA proximally extending to the EIA distally. Conduit dilation was performed with 6 and $8 \mathrm{~mm}$ balloons, which again permitted safe trans-iliac passage of an AUI graft to the main aortic body. The procedure was completed with a femoro-femoral crossover. The postoperative course was uncomplicated; however, she did require administration of long-term intravenous antibiotics in the community to limit the potential for superimposed graft infection. Follow-up imaging at 1 year revealed resolution of radiological signs of aortitis.

\section{Discussion}

The endoluminal conduit was first described by Yano et al in 2001. It offers an endovascular solution to unfavorable ilio-femoral anatomy [2]. Formerly, retroperitoneal open iliac conduit (ROIC) had been the primary mode of overcoming complex iliofemoral disease. This hybrid technique is associated with greater operative morbidity compared with standard approaches [5].

Yano et al describe incorporating a $6 \mathrm{~mm}$ endoluminal polytetrafluoroethylene conduit onto a Palmaz stent (Cordis; Johnson and Johnson Interventional Systems, Warren, NJ) and positioning the stent in the external iliac artery. The EIA is subsequently ballooned to $6-8 \mathrm{~mm}$ from within using a noncompliant balloon with minimal reported morbidity [2].

Hinchliffe et al, Peterson, and Vallabhaneni et al all describe similar techniques of successfully "paving and cracking" the iliac segment to secure proximal arterial access [6-9]. These techniques again involve relining and dilating stenosed iliac arteries with covered stents prior to the introduction of main body aortic stent [8]. Of note, neither Hinchcliffe et al, Peterson nor Vallabhaneni et al describe any operative complications. 
Most recently, van Bogerijen et al [10] presented results of the first comparative analysis of both ROIC and IE. This study included 39 patients undergoing thoracic EVAR. The authors noted fewer ilio-femoral complications with the endoconduit with no difference in 2-year survival. Furthermore, no difference was observed in claudication, limb loss or revascularization rates. They concluded that endoconduits were a safe and effective management option in well-selected patients.

Studies to date identify IE placement as a safe means of traversing challenging iliac disease. However, procedural morbidity including hematoma formation and claudication has been infrequently described [2,9]. Further concern involves the coverage of the hypogastric artery and hence the impairment of pelvic perfusion which may manifest as sexual dysfunction, buttock claudication, bowel ischemia or even paraplegia.

Thus, the use of IE in the emergency setting is infrequently described. We have found its use in emergency EVAR to be safe and feasible. With careful preoperative planning, this technique may potentially increase the number of aneurysms treatable via endovascular means particularly in a patient cohort often unfit for open intervention.

\section{Declarations}

None.

\section{Consent}

Both described patients have provided written consent for the writing and publication of this report.

\section{References}

1. Mohan PP, Rozenfeld M, Kane RA, Calandra JD, Hamb- lin MH. Nationwide trends in abdominal aortic aneurysm repair and use of endovascular repair in the emergency setting. J Vasc Interv Radiol. 2012;23(3):338-344.

2. Yano OJ, Faries PL, Morrissey N, Teodorescu V, Hollier LH, Marin ML. Ancillary techniques to facilitate endovascular repair of aortic aneurysms. J Vasc Surg. 2001;34(1):69-75.

3. Wu T, Carson JG, Skelly CL. Use of internal endoconduits as an adjunct to endovascular aneurysm repair in the setting of challenging aortoiliac anatomy. Ann Vasc Surg. 2010;24(1):114 e117-114 e111.

4. Murray D, Ghosh J, Khwaja N, Murphy MO, Baguneid MS, Walker MG. Access for endovascular aneurysm repair. J Endovasc Ther. 2006;13(6):754-761.

5. Lee WA, Berceli SA, Huber TS, Ozaki CK, Flynn TC, Seeger JM. Morbidity with retroperitoneal procedures during endovascular abdominal aortic aneurysm repair. J Vasc Surg. 2003;38(3):459-463; discussion 464-455.

6. Hinchliffe RJ, Ivancev K, Sonesson B, Malina M. "Paving and cracking": an endovascular technique to facilitate the introduction of aortic stent-grafts through stenosed iliac arteries. J Endovasc Ther. 2007;14(5):630-633.

7. Peterson BG, Matsumura JS. Internal endoconduit: an innovative technique to address unfavorable iliac artery anatomy encountered during thoracic endovascular aortic repair. J Vasc Surg. 2008;47(2):441-445.

8. Peterson BG. Conduits and endoconduits, percutaneous access. J Vasc Surg. 2010;52(4 Suppl):60S-64S.

9. Vallabhaneni R, Sorial EE, Jordan WD, Jr., Minion DJ, Farber MA. Iliac artery recanalization of chronic occlusions to facilitate endovascular aneurysm repair. J Vasc Surg. 2012;56(6):1549-1554; discussion 1554.

10. van Bogerijen GH, Williams DM, Eliason JL, Dasika NL, Deeb GM, Patel HJ. Alternative access techniques with thoracic endovascular aortic repair, open iliac conduit versus endoconduit technique. J Vasc Surg. 2014;60(5):11681176. 